\title{
One Time Stable below Knee Residual Limb in Pediatric Amputee
}

\author{
Wessal Al-Homaied*, Burhan Dhar and Zayed AlZayed \\ King Faisal Specialist Hospital and Research Centre, Kingdom of Saudi Arabia \\ *Corresponding author: Wessal Al-Homaied MD, Department of Orthopedics, Saudi Arabia
}

Submission: 鮆 February 01, 2018; Published: 阱 February 26, 2018

\begin{abstract}
Appositional or terminal overgrowth of bone is common in children with an acquired or congenital amputation. This problem is seen primarily after amputation through the diaphysis of the tibia. Management of this condition requires frequent prosthetic adjustments or operative revision of the limb. [1].

Fusion of the distal tibia and fibula in transtibial amputations was first described in the early $20^{\text {th }}$ century by Janos Ertl in Hungary. The concept was later popularized in the United States by Col.

Philip A. Deffer in the 1960s and multiple modifications of the original technique have since been described [2].

We concentrate on painless rounded functional residual limbs \& end weight bearing capability. So, we use Ertl's procedure to evaluate the effect of this procedure on termination of overgrowth of bone in two children, one diagnosed as bilateral tibia hemimelia and the other as bilateral vascular insufficiency both underwent bilateral transtibial amputation following that technique.
\end{abstract}

After reviewing the literature regarding that technique, our hospital is first in applying the modified Ertl's procedure in pediatric patients who need bilateral transtibial amputation due to pathological limbs.

Keywords: Tibiofibular bridge synostosis; Transtibial amputation; Modified ertl's procedure; Overgrowth

\section{Introduction}

Amputation surgery should be viewed by patient and surgeon as the next step toward reconstructive recovery. Surgically removing a nonfunctional distal extremity tissues and fashioning a competent and painless residual limb for ambulation is the ultimate goal.

Bony overgrowth is one of the major complications in skeletally immature amputee after transtibial amputation. Overgrowth stemming from terminal appositional, endosteal proliferation (rather than physeal growth), this overgrowth results from a combined process of periosteal and endosteal new bone formation and from terminal remodeling. There is also a phenomenon of traction, with the remaining periosteum becoming fixed to the bone at the end of the distal site and having no remaining control over diametric or latitudinal expansion. The end become progressively tapered because it fails to grow adequately in diametric fashion compared with the more proximal portions, although longitudinal growth (appositional: membranous, rather than endochondral) does occur. Osseous overgrowth manifests clinically as an increasing inequality in length between the stump (residuum) skeleton and its soft tissue covering. As this inequality increasing the end of the stump becomes tented over the sharp bone [3]. It is s characterized clinically by swelling, erythema, warmth, tenderness, formation of a bursa, and extreme cases perforation of the soft tissues by bone. Clinical management includes frequent prosthetic adjustments and operative revision of the limb, which are done at great financial cost to the health-care system and psychosocial cost to the child and family [4].

Proposed operative strategies for the treatment of overgrowth in a child who has had an amputation have included distal resection; proximal epiphyseodesis; capping of the distal medullary canal with a metallic, silicone, or polyethylene implant; transfer of cartilaginous, physeal, or cancellous bone; distal tibiofibular synostosis; and prolonged skin traction [4].

The osteomyoplastic lower extremity amputation procedure was described by Professor Janos v. Ertl, MD, in 1939. This encompassed the sum of his experiences from the post war eras, operating on an estimated 13,000 amputees. The principles of the surgical technique were not only limited to the amputation field but had their origins in the field of reconstructive surgery. The procedure arose from the observation that the periosteum had regenerative potential in bony injuries $[5,6]$ if periosteal tube not disturbed. Ertl 
first applied this principle to procedures utilizing osteo-periosteal grafts to the mandible and the skull during World War I. As this was a trench war, many soldiers survived with maiming injuries to their face and cranium. Ertl reconstructed these osseous defects with flexible, free osteo-periosteal grafts harvested from the tibia. As the potential for these grafts to regenerate an osseous structure was realized, the grafts were then applied to a wider use, including the spine, long bones, and amputations [5, 6].

In transtibial, attention is initially directed towards the tibia and fibula bones and fibular instability. Fibular instability is defined as excessive motion of the fibula bone relative to the tibia bone due to the actions of the musculo-tendinous attachments and lack of distal bony attachment (stability), leading to a "chop-sticking" effect of the two bones when walking with prosthesis. In turn, this leads to irritation of both soft tissue and nerves and rotational instability within the prosthesis. A flexible osteoperiosteal bone graft is elevated from the distal ends of both the tibia and fibula. The flexibility allows the leafs to be sutured together in tube-like fashion creating a synostosis, providing a bony framework which solidifies over time, stabilizing the fibula. In addition to creating a synostosis, the medullary canals of both bones is closed, re establishing a normal intramedullary pressure and improving the surrounding bone and soft tissue blood supply [7]. That allows the child to walk without bilateral prosthesis in urgent situation between bed and washroom at night.

Along with osseous reconstruction in amputations, particular attention was also applied to the handling of the soft tissues. Neuromuscular isolation, high ligation of the nerves, myoplasty, and smooth skin closure provided the patient with a rounded residual extremity with end-bearing capabilities. Ertl believed that this returned the residual extremity to as normal an anatomic, physiologic, and biologic state as possible.

KFSH \& RC is one of the biggest referral hospitals in the Middle East, and we saw a lot of patients who need an amputation of the nonfunctional distal extremity tissues. Many amputation surgeries have been performed, and we are facing the terminal overgrowth problem with increasing number of the cases. After reviewing the previous literatures regarding Ertl's procedure we applied it in two of our cases, bilateral limbs for each and we follow them to see their outcomes, the least follow up was one year.

\section{Patient Report}

First case: 4 year old, female, product of normal spontaneous vertical delivery, history of

maternal drug abuse, with congenital lower limb deformities diagnosed to have bilateral tibia hemimelia with non functional feet and ankles.

Second case: 3 year old, male, product of normal spontaneous vertical delivery, diagnosed to have vasculitis affecting his vascular supply of lower limbs ending up with gangrenous limbs with demarcated line just above the ankle.

\section{Discussion}

\section{Hospital course}

Both admitted to the hospital, informed consent was obtained from both patients, also seen by anesthesiologist pre operatively for assessment. And for both patients we follow the same operative steps following modified Ertl procedure.

\section{Steps of modified Ertl's procedure}

Following Ertl's procedure which described in John J. Keeling and Lew C. Schon, literature; (tibiofibular bridge synostosis in belowknee amputation) with some modifications from our side.

Patient was laying in a supine position, under tourniquet control. We followed the WHO check list for surgical patient. We also followed the known basic principles of transtibial amputation in skin incision and soft tissue dissection. After exposing the tibia and fibula, care was taken to avoid excessive fibular soft tissue stripping. The fibula was exposed two centimeters below the level of proposed tibial osteotomy.

Anterior and lateral compartment musculature transected at that level. Homan retractors were placed medial and lateral around the circumference of the fibula, and fibula was cut with an oscillating saw.

Similarly, tibia was osteotomized at the preplanned more proximal level. The anterior third of the tibia beveled at 30 degrees to prevent bony prominence postoperatively. Subperiosteal dissection was performed posteriorly, and then we lift the tibia and fibula away from the underlying musculature. An amputation knife was used to transect the posterior musculature and skin flap was one centimeter longer than the diameter of the leg at the tibial osteotomy level. The amputated distal limb was passed off as specimen.

A systematic anatomic dissection was then performed to isolate each peripheral nerve to help prevent painful postoperative neuroma formation. Each nerve was gently grasped, pulled distally, and transected as proximal as we can with a sharp scalpel. Similarly, the large arteries and their associated veins were individually identified and double suture ligated.

The lateral border of the tibia was now approached, and periosteal flaps are delicately created from the distal aspect of the bone to about two centimeters proximally. Then, we create a 4.5 millimeters hole by drill in that border distally. Next, we did a greenstick fracture in the fibula by two millimeters drill pit at the level of the tibia. With the medial fibula folded on its preserved periosteal medial hinge, a periosteal flap was created on the medial aspect of the fibula and dissected two centimeters proximal to the tibial level. The lateral musculature was left intact on the fibula segment with only partial detachment to permit rotation of the piece. Then the fibula was swung and inserted in the tibial hole that we created. If the fibula at the osteotomy site is weak, we tried to fix it in place with heavy nonabsorbable suture (Ethibone size one). 
The periosteal flaps were then sutured to the fibular segment to promote bony healing. The long posterior flap was brought anteriorly and fashioned with sharp transection of muscle and skin to the desired length for a tension-free closure without excessive redundancy. The tourniquet was released, and hemostasis was obtained. Hemovac drain was placed deep to the muscle.

Finally, the wound is closed in layers with a heavy nonabsorbable suture closing posterior muscle fascia to anterior fascia and bone (myoplasty) then subcutaneous closure then skin. The same was done for the other limb. Posterior slab applied for two weeks, wound inspected and non weight bearing cylindrical cast was applied for another four weeks, then the shrinker for other two weeks after that we use the transtibial prosthesis. Patient was fitted with the prosthesis in two months. The design of the prosthesis included a total tonact socket with equal weight bearing including the bottom of the residual limb. Patient was provided with the gait training with the prosthesis immediately after fitting of Prosthesis. Physical therapy was also instituted for education on walking, strengthening the quadriceps muscles and upper body and preventing fixed flexion deformity of the knee.

\section{Results}

In the previous literature, modified Ertl's procedure benefits the adult amputees in the following ways:

a. Prevention of excessive fibular motion by bony bridge (synostosis) stabilization in transtibial amputations.

b. Reconstruction of the medullary canal with reestablishment of intramedullary pressure and prevention of arteriovenous connections at the bone.

c. Improved venous return to the heart by myoplasty,

d. Prevention of nerve adhesion by restoring the gliding properties of the nerve. e. Improve prosthesis fitting with removal and prevention of unstable scars and creating a smooth contoured interface for prosthetic fitting.

f. Decreasing osteoporosis due to disuse atrophy by providing end bearing potential and reinstitution of physiological bone loading [7].

Due to the above benefits those patients are able to well balance and even take steps without prosthesis just like a symes amputee.

In our study of two patients, managed by modified Ertl osteomyoplasty, rapid synostosis of the fibula to the tibia occurred (Figure 1). There were no bony overgrowth complications. All the patients were fully ambulatory in their prostheses without pain; they can walk short distances with end weight bearing capabilities and even without wearing the prosthesis (Figure $2 \& 3$ ).

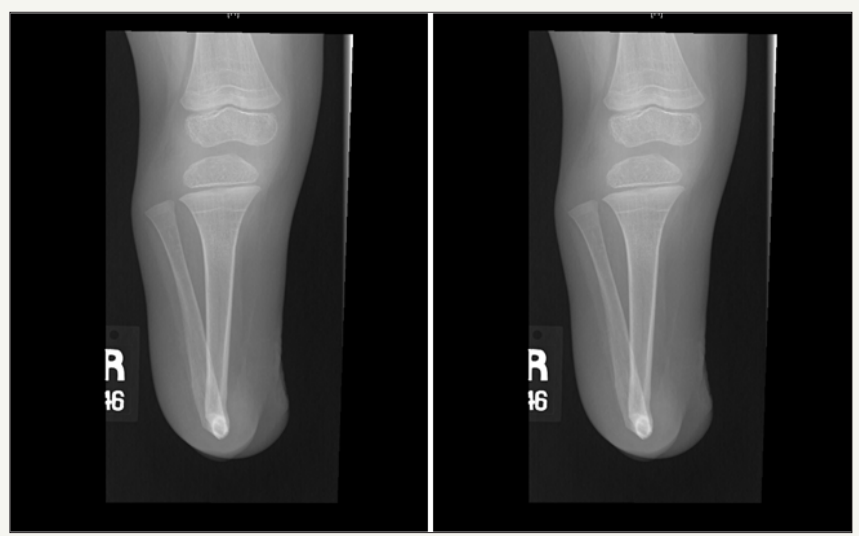

Figure 1: Show a PA and lateral views of a modified Ertl's procedure (transtibial amputation) of one of the patient showing a healed tibiofibular bony bridge.

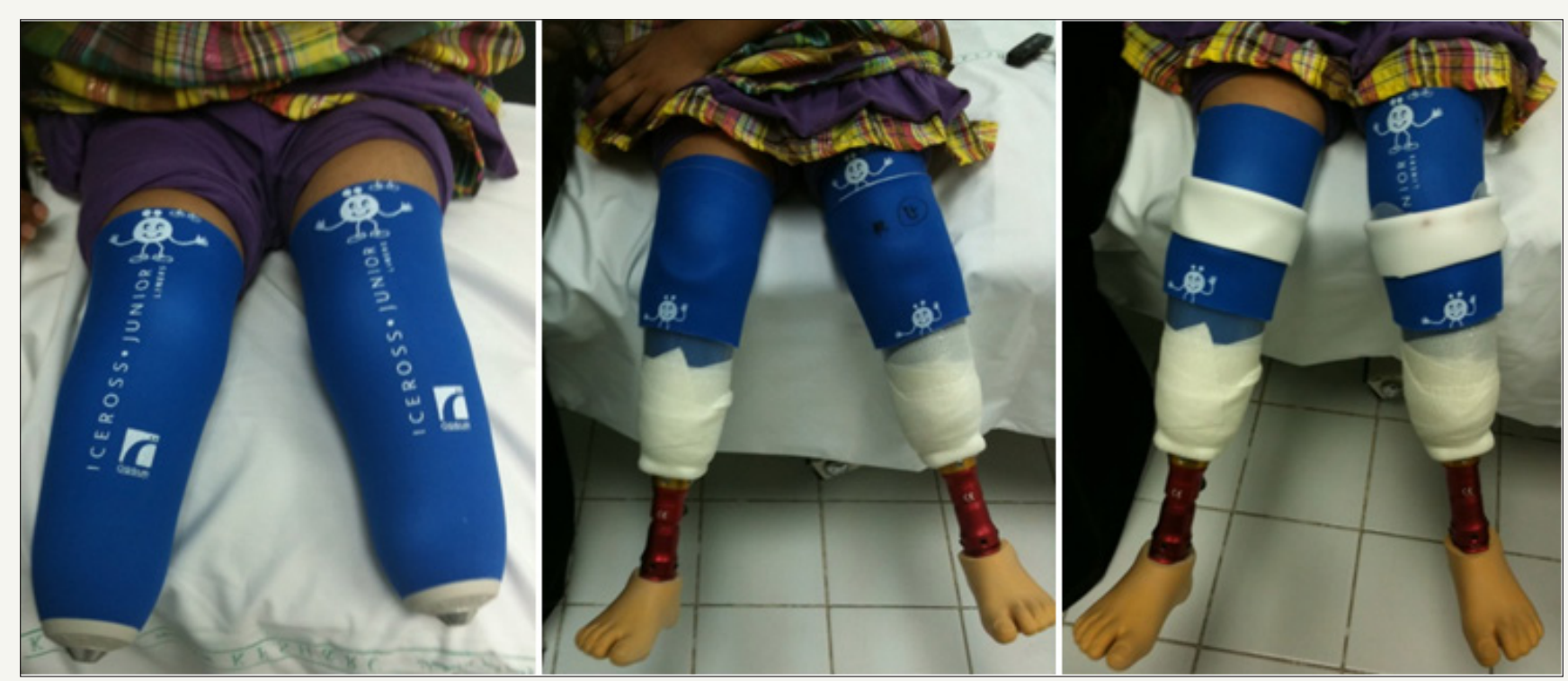

Figure 2 : show the first patient during her follow up. 


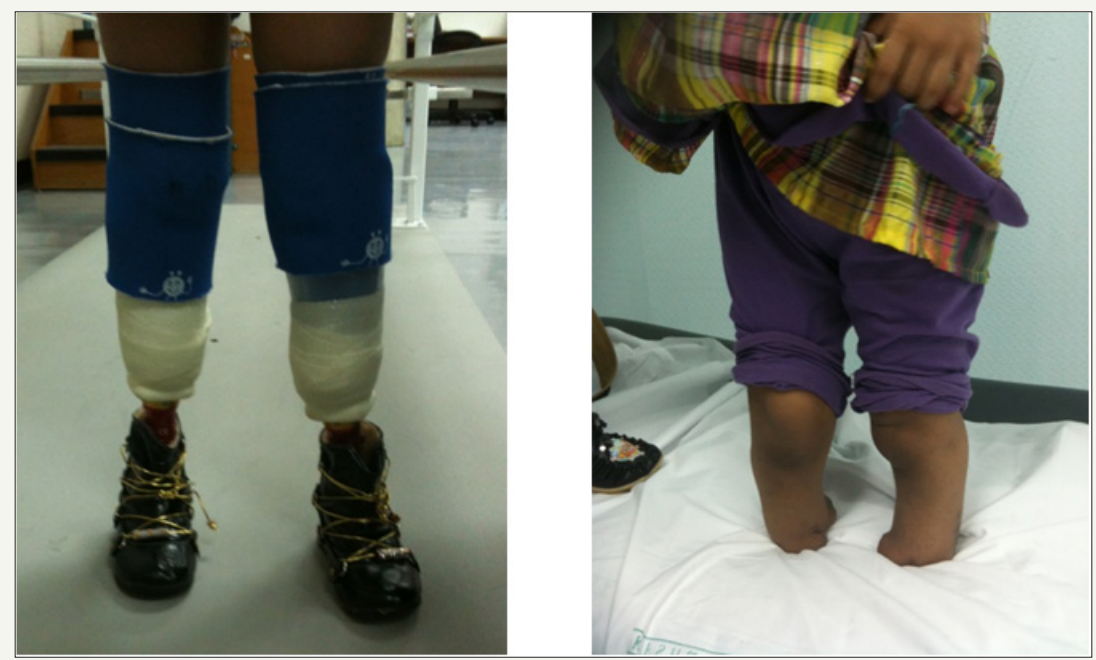

Figure 3 : show the first patient during standing with end weight bearing with and without wearing the prosthesis.

With no bony overgrowth although follow up is short we are not expecting bony overgrowth even with long follow up.

\section{Conclusion}

The osteomyoplastic transtibial amputation or reconstruction in pediatric transtibial amputee with bilateral pathology, Modified Ertl's procedure is technically challenging with more operative time needed than conventional techniques, but it has a high success potential and high patient satisfaction by preventing bony overgrowth and its complication regarding pain, instability \& prosthetic problems. Such amputations can lead to improved quality of life and thereby expand their horizon. We have therefore concluded that modified Ertl's procedure as one of the best solutions for transtibial amputation in children.

\section{References}

1. David M, Drvaric MD, Leon M kruger (2001) Modified Ertl osteomyoplasty for terminal overgrowth in childhood limb deficiencies. Study conducted at the shriners hospital for children, springfield, Massachusetts, USA.
2. Jaymes DG, Terrence M, Philbin (2010) Distal tibiofibular bone bridging in transtibial amputation. Current Orthopaedic Practice 21(3): 264-267.

3. Ogden JA (1982) Skeletal injury in the child. ( $3^{\text {rd }}$ edn), Springer-Verlag New York, USA, p. 295.

4. Davids JR, Meyer LC, Blackhurst DW (1995) Operative treatment of bone overgrowth in children who have an acquired or congenital amputation. J Bone Joint Surg Am 77(10): 1490-1497.

5. von Johann V, Barth A, Leipzig (1939) Regeneration: Ihre Anwedung in der Chirurgie.

6. Ertl JP, Barrack R, Alexander AH, VanBuecken KP (1988) Triplane fracture of the distal tibial epiphysis. Long Term Follow-up. J Bone Joint Surg 70(7): 967-976.

7. Jan Ertl (2000) Pain and the inactive residual extremity syndrome. 10(5).

\section{Your subsequent submission with Crimson Publishers will attain the below benefits}

- High-level peer review and editorial services

- Freely accessible online immediately upon publication

- Authors retain the copyright to their work

- Licensing it under a Creative Commons license

- Visibility through different online platforms

- Global attainment for your research

- Article availability in different formats (Pdf, E-pub, Full Text)

- Endless customer service

- Reasonable Membership services

- Reprints availability upon request

- One step article tracking system 